\title{
Dust and Radiation Quantum Perfect Fluid Cosmology : Selection of Time Variable
}

\author{
I-Chin Wang* \\ Department of Physics, National Cheng Kung University \\ Tainan, Taiwan 701
}

(Dated: August 7, 2018)

\begin{abstract}
We studied the expectation value of the scale factor in radiation and dust quantum perfect fluid cosmology. We used Schutz variational formalism to describe perfect fluid and selected the conjugate coordinate of perfect fluid be dynamical variable. After quantization and solving the WheelerDeWitt equation can obtain the exact solution. By superposition of the exact solution, we obtained one wave packets and used it to compute the expectation value of the scale factor. We found that if one select different dynamical variable be the time variable in each of these two systems, the expectation value of the scale factor of these two systems can fit in with the prediction of General Relativity. Therefore we thought that the selection of reference time can be different for different quantum perfect fluid systems.
\end{abstract}

PACS numbers: 98.80.Jk, 98.80.Qc

\section{INTRODUCTION}

It is known that time disappear in the canonical quantization based on Arnowitt-Deser-Misner(ADM) decomposition. The notion of time can be recovered in some case of quantum cosmology, for example when gravity were coupled to a perfect fluid $[3,4,[6]$. These kind of systems were often dealt with in the following way (see [4, 6$]$ ): First they used Schutz's formalism for the description of perfect fluid [1], second they selected the dynamical variable of perfect fluid be reference time. Then used canonical quantization to obtain the Wheeler-Dewitt equation in minisuperspace, which is a Schrödinger like equation [3]. After solving the equation and choosing a weighting, they can obtain one wave packets. The obtained wave packets can be used to compute the expectation value of the scale factor. By using the wave packets they obtained the time-dependent behavior of the scale factor. If the selected time variable(dynamical variable of perfect fluid) can let the expectation value of the scale factor fit in with the classical prediction(prediction of General Relativity) when time is large, the selected time variable can be viewed as a good time variable for the system.

The above method can reconstruct a time variable and let the obtained expectation value fit in with GR's prediction, but there are still some questions. First, although the solution for both the radiation-dominated quantum PF(perfect fluid) system and the dust-dominated quantum PF system can be obtained, the good weighting function is hard to find. In order to avoid this problem, Lemos [7] proposed another procedure. He chosen a special initial wave packets satisfied the dynamical equation of the system. Then he used the wave packets and the propagator to compute the dynamical evolution of the scale factor, the behavior of the scale factor did fit in with classical prediction.

Although this problem had been solved by Lemos but there is another way to discuss this problem. In this article we try to discuss these two systems in the other way-by choosing a weighting and a suitable time variable. We obtained the exact solution of radiation-dominated and dust-dominated quantum PF cosmology. We found that for the radiation-dominated system, if the square of the perfect fluid dynamical variable can be treated as time variable, there exist a suitable weighting function that can reconstruct a wave packets which make the expectation value of the scale factor fit in with classical prediction. While for the dust-dominated quantum PF cosmology, there exist another suitable weighting function which make the perfect fluid dynamical variable be a good time variable. In other words, different weighting function corresponds to different choice of time variable.

This paper is organized as follows. In the next section, the quantum perfect fluid cosmological model in minisuperspace was established. By using Schutz's formalism, the Wheeler-DeWitt equation for arbitrary barotropic equation of state $p=\alpha \rho$ is written down. In section 3, we solved the exact solution for radiation and dust universe in flat case. After choosing a weighting function, we obtain the wave packets. Then we use the wave packets to compute the expectation value of the scale factor of universe. We found that the time variable and the weighting function can be chosen differently for these two different system. For dust-dominated system, the perfect fluid dynamical variable is

*Electronic address: icwang@hepth.phys.ncku.edu.tw 
a good time variable. While for radiation-dominated system, the square of the perfect fluid dynamical variable is a suitable time variable. Section 4 is discussion.

\section{THE QUANTUM MODEL}

The action for a perfect fluid coupled to gravity in Schutz's formalism can be described as

$$
S=\int_{M} d^{4} x \sqrt{-g} p-\int_{M} d^{4} x \sqrt{-g} R-\int_{\partial M} d^{3} x \sqrt{h} K
$$

where $\mathrm{p}$ is the pressure, $\mathrm{K}$ is the trace of the extrinsic curvature and $\mathrm{h}$ is the induced metric in the boundary of the manifold M. The pressure $\mathrm{p}$ is linked to the energy density by the equation of state $p=\alpha \rho$. The four velocity of the fluid is expressed in terms of the five potentials $\epsilon, \phi, \beta, \theta$ and $S$ :

$$
u_{\nu}=\frac{1}{\mu}(\epsilon, \nu+\phi \beta, \nu+\theta S, \nu)
$$

where $\mu$ is the specific enthalpy. The four velocity is subjected to the condition $u^{\nu} n_{\nu}=1$. We consider the FriedmannLemaitre-Robertson-Walker metric

$$
d s^{2}=-N^{2} d t^{2}+a^{2}(t) \sigma_{i j} d x^{i} d x^{j}
$$

the super-hamiltonian can be constructed as $3,4,4,6]$

$$
H=-\frac{\Pi_{a}^{2}}{24 a}-6 k a+\frac{e^{S} p_{\epsilon}^{1+\alpha}}{a^{3 \alpha}}
$$

where $\alpha$ was mentioned above as the equation of state $(p=\alpha \rho)$. Performing the canonical transformations [3, [4, 6]

$$
T_{2}=-p_{s} e^{-s} p_{\epsilon}^{-(1+\alpha)} \quad, \quad \Pi_{T_{2}}=e^{s} p_{\epsilon}^{1+\alpha}
$$

where $T_{2}$ is the dynamical variable for perfect fluid, $\Pi_{T_{2}}$ is the conjugate momentum of $T_{2}$. The super-hamiltonian can be written as

$$
H=-\frac{\Pi_{a}^{2}}{24 a}-6 k a+\frac{\Pi_{T}}{a^{3 \alpha}}
$$

note that $\Pi_{T_{2}}$ is also the momentum associated with the matter variable, $k$ represents the curvature of the 3 -space with the values $0,1,-1$ for a flat, closed and open Universe. Introducing the quantization conditions to the hamiltonian, we obtain the Wheeler-DeWitt equation in the minisuperspace:

$$
\frac{\partial^{2} \Psi}{\partial a^{2}}-144 k a^{2}-i 24 a^{1-3 \alpha} \frac{\partial \Psi}{\partial T_{2}}=0
$$

the equation (6) is equivalent to the Schrödinger equation of ordinary quantum mechanics. Thus, all the formalism of quantum mechanics can be applied to this problem. Applying the separation of variables method, writting

$$
\Psi(a, T)=e^{i E T} \psi(a)
$$

we obtain

$$
\varphi^{\prime \prime}+\left(-144 k a^{2}+24 E a^{1-3 \alpha}\right) \psi=0
$$

where the primes mean derivative with respect to a. So far, we obtain the equation for 3-space. After defining the curvature of the 3 -space and the matter which is filled in this Universe, we will obtain the dynamical equation for the individual Universe.

\section{BEHAVIOR OF THE SCALE FACTOR WITH THE SELECTION OF TIME VARIABLE}

We consider the flat case $(\mathrm{k}=0)$. Thus equation $(9)$ reduces to

$$
\psi^{\prime \prime}+24 E a^{1-3 \alpha} \psi=0
$$

Although the general solution of equation (10) has been obtained [4, 6], but this general solution can not become radiation-dominated solution when $\alpha=\frac{1}{3}$. We first consider radiation-dominate case. 


\section{A. Radiation $(\alpha=1 / 3)$}

For $\alpha=\frac{1}{3}$, equation (10) reduced to

$$
\psi^{\prime \prime}(a)+24 E \psi(a)=0
$$

the solution is

$$
\psi(a)=D_{1} \sin (\sqrt{E} a)+D_{2} \cos (\sqrt{E} a)
$$

note that the solution is not the form of Bessel function. We choose $D_{1}=0$ to construct wave packets, and our boundary condition [7] for wave packets is

$$
\left.\frac{\partial \Psi\left(a, T_{2}\right)}{\partial a}\right|_{a=0}=0
$$

put $\psi(a)=D_{2} \cos (\sqrt{E} a)$ into equation (8). To obtain a wave packets, we choose a weighting function $W(E)$

$$
\Psi\left(a, T_{2}\right)=\int_{0}^{-\infty} e^{i E T_{2}} \cos (\sqrt{E} a) W(E) d E
$$

where $W(E)$ is the weighting. Let $E=y^{2}$ and take $W(y)=y^{-1} e^{-\lambda y^{2}}$, then put these into equation (12)

$$
\Psi\left(a, T_{2}\right)=\int_{0}^{-\infty} 2 e^{-\left(\lambda-i T_{2}\right) y^{2}} \cos (y a) d y
$$

note that $\operatorname{Re} \lambda>0$, use formula [8] to get the wave packets

$$
\Psi\left(a, T_{2}\right)=-\sqrt{\frac{\pi}{\left(\lambda-i T_{2}\right)}} \exp \left\{-\frac{a^{2}}{4\left(\lambda-i T_{2}\right)}\right\}
$$

the inner product must take the form in order for the Hamiltonian operator $\hat{H}$ to be self-adjoint

$$
(\Phi, \Psi)=\int_{0}^{\infty} a^{1-3 \alpha} \Phi^{*} \Psi d a
$$

the expectation value of the scale factor is

$$
<a>\left(T_{2}\right)=\frac{\int_{0}^{\infty} \Psi^{*}\left(a, T_{2}\right) a \Psi\left(a, T_{2}\right) d a}{\int_{0}^{\infty} \Psi^{*}\left(a, T_{2}\right) \Psi\left(a, T_{2}\right) d a}
$$

leading to

$$
<a>\left(T_{2}\right)=\frac{\sqrt{\lambda^{2}+T_{2}^{2}}}{\sqrt{2 \lambda \pi}} \propto T_{2}
$$

Now we choose $T_{2}=\sqrt{T}$, that $T$ is our time variable. When $T \rightarrow \infty$ the expectation value of $\langle a\rangle$ becomes

$$
<a>(T) \propto T^{\frac{1}{2}}
$$

the behavior of the scale factor for radiation is proportional to half order of $T$, just fit in with classical prediction. In this case with this weighting function $W(y)$, we see that $T_{2}^{2}$ is the suitable time variable. Next we discuss dustdominated system.

\section{B. Dust $(\alpha=0)$}

The solution of equation (9) for $\alpha=\frac{1}{3}$ had been obtained [4, 6, 7]. We review this dust-dominated system to see which dynamical variable could be time variable. 
For $\alpha=0$, equation (9) reduce to

$$
\psi^{\prime \prime}(a)+24 \operatorname{Ea} \psi(a)=0
$$

the solution is the form of Bessel function

$$
\psi(a)=\sqrt{z}\left\{c_{1} J_{\frac{1}{3}}\left(\frac{2}{3} z^{\frac{3}{2}}\right)+c_{2} J_{\frac{-1}{3}}\left(\frac{2}{3} z^{\frac{3}{2}}\right)\right\}
$$

where $z=-E^{\frac{1}{3}} a$

$$
\Psi_{E}=e^{i E T_{2}} \sqrt{z}\left\{c_{1} J_{\frac{1}{3}}\left(\frac{2}{3} z^{\frac{3}{2}}\right)+c_{2} J_{\frac{-1}{3}}\left(\frac{2}{3} z^{\frac{3}{2}}\right)\right\}
$$

we choose $c_{2}=0$ and a weighting function $W^{\prime}(E)$ to construct the wave packet, the boundary condition for the wave packets is the same as e.q.(13)

$$
\Psi\left(a, T_{2}\right)=\int_{0}^{-\infty} W^{\prime}(E) \Psi_{E}\left(a, T_{2}\right) d E
$$

let $E=y^{2}, W^{\prime}(y)=e^{-\lambda y^{2}}($ note $\operatorname{Re} \lambda>0)$ and use formula [9] , the wave packets is

$$
\Psi\left(a, T_{2}\right)=\frac{c_{4}}{\left\{2\left(\lambda-i T_{2}\right)\right\}^{\frac{4}{3}}} \exp \left\{\frac{-c_{3}^{2}}{4\left(\lambda-i T_{2}\right)}\right\}
$$

where $c_{3}=-\frac{2}{3} a^{\frac{3}{2}}, c_{4}=\frac{2}{3} c_{1}(1-i \sqrt{3}) a$, the expectation value of the scale factor (for $\alpha=0$ ) is

$$
<a>\left(T_{2}\right)=\frac{\int_{0}^{\infty} a \Psi^{*}\left(a, T_{2}\right) a \Psi\left(a, T_{2}\right) d a}{\int_{0}^{\infty} a \Psi^{*}\left(a, T_{2}\right) \Psi\left(a, T_{2}\right) d a} \propto\left(\lambda^{2}+\frac{T_{2}^{2}}{\lambda}\right)^{\frac{1}{3}}
$$

when $T_{2} \rightarrow \infty$ :

$$
<a>\left(T_{2}\right) \propto T_{2}^{\frac{2}{3}}
$$

Thus for dust-dominated quantum cosmology $(\alpha=0)$ with the weighting function $W^{\prime}(y), T_{2}$ ( the dynamical variable for dust perfect fluid ) is a suitable time variable because it makes the behavior of scale factor fit in with classical prediction.

Thus we can see that the suitable time variable depends on the weighting function we chosen.

\section{DISCUSSION}

If we think that time disappear in the canonical quantization based on Arnowitt-Deser-Misner(ADM) decomposition can be solved by choosing a dynamical variable as the time variable, then we can find that the choice is not unique. In section 3, we can see that a suitable time variable depends on the weighting function one used.

Note that in Lemos's paper, the situation is different. Lemos chosen a suitable wave packets to satisfy his time variable (the dynamical variable for perfect fluid). In other words, he first decided the time variable of the system then search a good wave packets to make the behavior of the scale factor fit in with classical prediction.

Therefore in the canonical quantization based on ADM decomposition, the choice of time variable is not unique but is relative. The wave packets we used did affect the time variable we chose. In this point of view, time is not an absolute coordinate, it is a relative parameter.

As for the parameter $t$ appeared in the equation (3), we consider that $t$ is an introduced parameter which helps us to think of a physical system. We think that this is the case Barbour et al proposed [10]. In the article there are two sentence [11]:

The theory of time and clocks must be derived from the dynamics of the universe. The fact is that speed is not obtained by dividing an infinitesimal displacement by an infinitesimal time but by another displacement. 
Thus we think that time variable can be chosen and in the canonical quantization based on ADM decomposition, it depends on the wave packets we used.

[1] B. F. Schutz, Phys. Rev. D2 (1970) 2762 ; Phys. Rev. D4 (1971) 3553 .

[2] Robert M. Wald, GENERAL RELATIVITY, The University of Chicago Press , p98 .

[3] V. G. Lapchinskii and V. A. Rubakov, Theor. Math. Phys. 33 (1977) 1076.

[4] F.G. Alvarenga, J.C. Fabris, N.A. Lomes and G.A. Monerat, Gen. Rel. Grav. 34 (2002) 651.

[5] for example let $\alpha=\frac{1}{3}$ in equation(22) of Gen. Rel. Grav. 34 (2002) 651 .

[6] A.B. Batista , J.C. Fabris , S.V.B. Gonçalves and J. Tossa , Phys. Rev. D65 (2002)063519 .

[7] N.A. Lemos, J. Math. Phys. 37(1996)1449 .

[8] formula 3.896-4, Table of Integrals, Series and Products ,ACADEMIC PRESS .

[9] formula 6.631-4, Table of Integrals, Series and Products ,ACADEMIC PRESS .

[10] Julian Barbour, Bredan Z. Foster and Niall ó Murchadha, Class. Quantum Grav. 19 (2002) 3217 and gr-qc/0012089

[11] page 3 of reference [10]. 